\title{
Information et énergie nucléaire
}

\author{
(Manuscrit reçu le 5 mai 1982)
}

\author{
H. JOFFRE (*)
}

\begin{abstract}
RÉSUMÉ
Les documents établis par les organismes internationaux et nationaux apportent une information très complète sur les aspects sécurité dans le domaine de l'énergie nucléaire.

Relativement aux nuisances chimiques ou biologiques, la détection des rayonnements est particulièrement simple et sensible.

Les effets biologiques des rayonnements ionisants sont bien connus grâce aux enquêtes épidémiologiques et à l'expérimentation biologique. Des relations entre doses et effets biologiques ont été précisées qui permettent une évaluation des risques associés à toute exposition aux rayonnements ionisants : irradiation naturelle, utilisation du rayonnement $X$ en radiodiagnostic, production d'énergie nucléaire,...

Une étude comparative montre que les risques globaux associés à la production d'électricité d'origine nucléaire sont beaucoup plus faibles que ceux liés à la production d'énergie électrique par les combustibles fossiles, ces derniers risques etant eux-mêmes beaucoup plus faibles que ceux résultant de l'usage du tabac.
\end{abstract}

\section{ABSTRACT}

Comprehensive information on the safety aspects in the field of nuclear energy is supplied by the documents established by international and national bodies. As compared with chemical or biological hazards, the detection of radiation is especially simple and sensitive. The biological effects of ionizing radiation are well known thanks to epidemiological surveys and biological experiments. The dose-effect relationships have been determined allowing an assessment of the risks associated with any radiation exposure : natural exposure, $\mathrm{X}$-ray in medical diagnosis, nuclear energy production,...

A comparative study shows that the overall risks associated with the production of electric energy of nuclear origin are lower than those linked to fossil-fueled power plants, the latter being much lower than the risks from smoking.

(*) Commissariat à l'énergie atomique, Institut de protection et de sûreté nucléaire, Département de protection, BP 6, 92260 Fontenay-aux-Roses. 


\section{QUELQUES REMARQUES SUR L'INFORMATION DU PUBLIC DANS LE DOMAINE NUCLÉAIRE}

\section{I.1. Généralités}

Tout comme dans de nombreux domaines scientifiques, beaucoup font confiance aux chercheurs et aux ingénieurs qui contribuent au développement de l'énergie nucléaire. Pour d'autres, au contraire, il existe une grande inquiétude qui est cultivée et développée par de nombreux articles de presse dont le but est plus souvent d'inquiéter le lecteur que de l'informer.

Parvenir à faire assimiler par l'opinion publique une information objective dans un domaine scientifique et technique est un problème difficile. Déjà, ceux qui sont chargés de l'administration de leur commune ou de leur département et qui ont de larges possibilités d'information ressentent souvent la difficulté de parvenir à une compréhension correcte de la sécurité nucléaire. Ces personnalités doivent néanmoins s'informer avec persévérance afin de parvenir à ce résultat. Même si cette tâche est difficile, elle reste un devoir de tout responsable.

L'information disponible concernant les niveaux de rayonnements naturels ou résultant des activités humaines civiles et militaires, les effets biologiques des rayonnements, les moyens de protection et de sûreté nucléaires, est très complète et très précise (voir les documents établis par des experts d'organismes internationaux ou nationaux: Nations Unies (ONU), Commission internationale de protection radiologique (CIPR), Agence internationale de l'énergie atomique (AIEA), National Academy of Sciences (aux USA),... $[4,7,8]$, mais elle est aussi d'un niveau scientifique indispensable à une présentation objective des problèmes.

Vulgariser un tel sujet et le présenter en une ou même quelques conférences ne peut pas prétendre constituer une information suffisante : celui qui veut être informé, et qui doit l'être en raison de ses responsabilités, doit s'astreindre à une recherche approfondie en la matière.

\section{I.2. Connaissance et mesure des rayonnements}

Les rayonnements sont connus et utilisés par l'homme depuis plus de 80 ans ; ils ne constituent pas un phénomène nouveau et les risques associés sont également bien connus, surtout si la comparaison est faite avec nos connaissances sur les risques associés aux innombrables produits chimiques cancérigènes.

Un premier aspect qui caractérise les rayonnements est la possibilité de les détecter avec une sensibilité très élevée devant la dose nécessaire pour produire un effet biologique décelable. En outre, un nombre très limité de détecteurs est suffisant pour assurer la détection de tous les rayonnements ionisants.

Ces caractéristiques particulières permettent de parvenir à une très grande sécurité pour les travailleurs qui sont immédiatement informés d'une faible variation du niveau d'irradiation ; par contre, il n'existe pas beaucoup de cas, dans l'industrie chimique par exemple, où les travailleurs sont 
en permanence tenus informés du niveau de pollution de l'air et de ses variations par une signalisation lumineuse et sonore. Ces dispositions, possibles et souvent appliquées dans le domaine nucléaire, permettent de limiter considérablement les expositions des travailleurs en cas d'accident : un risque de contamination de l'air par du plutonium est ainsi infiniment moins sournois et aussi moins grave de conséquences que s'il s'agissait de béryllium, d'amiante, de bacilles ou de virus pathogènes pour lesquels la détection instantanée associée à une signalisation immédiate n'est pratiquement pas réalisable.

La sensibilité de détection des rayonnements est très grande : Un détecteur de poche à tube-compteur Geiger-Müller permet la détection rapide d'une radioactivité ambiante même faible : le niveau d'irradiation naturelle, soit environ 1 cent-millième de rem par heure, produit un taux de comptage d'environ 100 impulsions par minute (par contre, un niveau d'irradiation dans le faisceau d'un générateur $X$ de 100 rems par minute conduirait à un taux de comptage 600 millions de fois plus élevé si le compteur n'était pas saturé avant).

\section{I.3. Connaissance des effets biologiques des rayonnements}

L'induction de cancers et de leucémies par les rayonnements est aujourd'hui relativement bien connue grâce aux enquêtes épidémiologiques $[7,8]$ qui ont porté sur:

- les expositions professionnelles (plusieurs milliers de personnes) : radiologues, travailleurs des mines d'uranium, peintres de cadrans lumineux;

- les expositions à des fins médicales (plusieurs dizaines de milliers de personnes) : radiothérapie de la spondylarthrite ankylosante, radiodiagnostic (pelvimétries, examens pulmonaires, utilisation du thorotrast,...) ;

- les expositions des survivants irradiés à Nagasaki et à Hiroshima (45000 personnes irradiées entre 1 et 600 rem).

Les effets ainsi observés pour des doses délivrées à des débits élevés ont permis d'établir une limite supérieure du risque associé à une dose unitaire de rayonnement.

En outre, l'application générale, par prudence, d'une relation doșe-effet linéaire et sans seuil (nombre de cancers induits proportionnel à la dose) constitue un deuxième facteur de sécurité au moins pour ce qui concerne les rayonnements peu ionisants, tels que les rayonnements $\beta, \gamma$ et les muons cosmiques pour lesquels une relation quadratique (nombre de cancers induits proportionnel au carré de la dose) paraît plus probable. Pour ces rayonnements très peu ionisants, le facteur de sécurité, résultant de l'utilisation d'une relation linéaire pour extrapoler aux doses inférieures à $1 \mathrm{rem}$ les effets constatés à des doses et à des débits élevés, peut ainsi être supérieur à 10 .

Si des progrès restent à accomplir pour une connaissance plus précise des effets biologiques des rayonnements ionisants, il n'en demeure pas moins que les méthodes d'évaluation des risques dus aux rayonnements constituent aujourd'hui un modèle pour l'étude des effets cancérigènes des substances chimiques où quasiment tout est à faire.

L'application, dans les conditions définies par la publication 26 de la CIPR, [4], des limites de dose pour toute exposition aux rayonnements en général ou des limites d'incorporation annuelle par inhalation ou ingestion 
de radionucléides constitue une disposition pratique très efficace contre l'induction de cancers et d'effets génétiques éventuels. Par contre, les valeurs analogues fixées dans le domaine de la pollution chimique, et encore pour un nombre très limité de polluants, ne constituent en général qu'une protection contre l'intoxication aiguë et non contre l'induction de cancers pourtant tout aussi importante que dans le domaine des rayonnements ionisants.

\section{SIGNIFICATION DU MOT "ACCIDENT" DANS LE DOMAINE NUCLÉAIRE}

\section{II.1. Exposition accidentelle}

Compte tenu des connaissances acquises dans le domaine des effets biologiques des rayonnements, l'application, dans les conditions définies par la CIPR, de la limite de dose annuelle pour les travailleurs rend les risques éventuels dus aux rayonnements inférieurs à ceux liés aux autres activités professionnelles, dans l'hypothèse pessimiste où ces travailleurs seraient exposés durant la totalité de leur carrière professionnelle [4].

Toute exposition ayant entraîné, en une période de 12 mois, une dose atteignant la limite annuelle, soit environ $2 \%$ de la limite d'exposition pour la totalité de la carrière professionnelle, fait l'objet d'une déclaration d'exposition accidentelle aux rayonnements ionisants à l'Inspecteur du travail et au Service central de protection contre les rayonnements ionisants (SCPRI) du ministère de la santé publique. Les conséquences, de toute évidence très faibles, d'une telle dose font que cette exposition accidentelle n'est pas ici synonyme de morbidité et encore moins de mortalité.

En outre, afin d'améliorer la sécurité du travail, le degré de surveillance habituellement appliqué dans les établissements nucléaires conduit souvent à procéder à l'examen, au sein de commissions spécialisées, des conditions d'exposition fortuite ayant entraîné des doses qui sont très inférieures à la limite de déclaration indiquée ci-dessus.

\section{II.2. Dossier de maladie professionnelle}

La législation fait obligation au médecin du travail de déclarer une maladie comme maladie professionnelle dès lors qu'elle figure sur le tableau de référence et que le travailleur est affecté à un poste de travail en présence de rayonnements, même si les doses effectivement subies, du fait des activités professionnelles, sont très faibles ou mêmes nulles.

Sur une population de 10000 personnes, et indépendamment de toute cause professionnelle, il y aura au total environ 2100 décès par cancer, soit, pour une longévité moyenne de 70 ans, environ 30 décès par cancer chaque année.

Compte tenu de ce qui précède, et même en l'absence d'exposition aux rayonnements, il est évident qu'un certain nombre de dossiers pour maladie professionnelle seront, à la suite de décès par cancer ou leucémie, communiqués à la Sécurité sociale. Or, il est courant de lire dans la presse que tel décès par cancer ou leucémie d'un travailleur d'un éta- 
blissement nucléaire résulte de la dose de rayonnement subie. Les contrôles effectués montrent, en fait, que la dose subie professionnellement est très souvent inférieure à l'irradiation naturelle moyenne de 0,1 rem par an.

Afin d'éviter de porter un tel jugement préremptoire, il paraît utile de rappeler, en outre, les points suivants:

a) Dans l'hypothèse d'une relation dose-effet linéaire (hypothèse acceptable dans le cas des rayonnements $\alpha$, des neutrons et des rayonnements X), il est admis que la probabilité de mortalité par cancer est de 1/10000 par rem ; en d'autres termes, on admet qu'il apparaîtra, en moyenne, un cancer mortel pour 10000 personnes exposées à 1 rem.

b) La dose moyenne due au rayonnement $X$ utilisé pour le radiodiagnostic médical est d'environ 0,1 rem par an.

c) La dose moyenne au niveau des poumons, résultant de la radioactivité naturelle $\alpha$ de l'air (due pour plus de $90 \%$ au polonium 214, produit de filiation du radon 222) présente un risque équivalent à une irradiation de l'organisme entier proche de 0,1 rem par an.

d) La dose moyenne d'irradiation de l'organisme entier résultant des rayonnements naturels peu ionisants (rayonnements $\gamma$ telluriques, muons cosmiques et rayonnements $\beta, \gamma$ du potassium 40 fixé dans l'organisme humain) est d'environ 0,1 rem par an.

e) Dans le cas des rayonnements peu ionisants, tels que les rayonnements $\beta, \gamma$, qui constituent l'essentiel de l'exposition associée à l'énergie nucléaire, les effets observés [7, p. 259] paraissent très inférieurs aux effets théoriques définis au paragraphe a). L'application d'une relation doseeffet linéaire conduit, dans ce cas, à une évaluation du risque qui est probablement très supérieure à la réalité.

Compte tenu des considérations ci-dessus, on ne peut jamais affirmer systématiquement que tout décès d'un travailleur par cancer résulte d'une exposition professionnelle, surtout lorsque cette exposition s'avère être plus faible ou de même ordre que l'exposition non professionnelle évaluée sur plusieurs décennies. Seule une estimation statistique portant sur un grand nombre de personnes ayant subies des expositions notables est susceptible de faire apparaître un effet des rayonnements. En effet, indépendamment de toute cause professionnelle, la fréquence des décès par cancers est d'environ $21 \%$. A titre d'exemple, cette fréquence pourrait être portée à $22 \%$ pour une population dont chaque individu aurait subi une exposition accidentelle de 100 rems. Elle serait, par contre, réduite à moins de $16 \%$ par suppression de l'usage du tabac ou, au contraire, accrue à plus de $30 \%$ par la généralisation de cet usage.

\section{POLLUTION DE L'AIR RÉSULTANT DE LA PRODUCTION D'ÉNERGIE ÉLECTRIQUE}

\section{III.1. Généralités}

Indépendamment des avantages économiques indiscutables de l'énergie nucléaire, actuellement et encore pour plusieurs décennies (coût du kWh nucléaire 3 fois moins cher que le kWh pétrole et 2 fois moins cher que le kWh charbon), il faut considérer les risques biologiques associés aux différents modes de production d'énergie. 
Aucune production d'énergie n'est sans risque et sans nuisance. Pour ce qui concerne les risques d'induction de cancers et de leucémies, une comparaison doit impérativement être prise en considération pour chacun des modes de production d'énergie électrique utilisant l'uranium, le pétrole et le charbon; ce n'est qu'au vu de cette comparaison qu'un jugement objectif peut être porté. Ces risques existent au niveau de l'extraction de la matière première, de l'exploitation des centrales et de l'élimination des effluents et déchets.

Sans entreprendre une étude complète de tous ces facteurs, on peut indiquer les précisions suivantes.

\section{III.2. Pollution radioactive}

Les risques associés à l'extraction du minerai d'uranium résident principalement dans l'exposition aux radionucléides naturels que sont le radon et surtout ses produits de filiation radioactive. Cette exposition par inhalation est du même type que celle subie dans les locaux d'habitation du fait de la radioactivité naturelle (qui existe partout et qui est présente dans tous les matériaux de construction) où elle atteint une valeur moyenne de 0,6 rem par an au niveau des poumons; cette valeur pouvant être multipliée par 10 dans le cas des locaux d'habitation très peu ventilés [8].

Dans les galeries de mines d'uranium, l'exposition des mineurs est maintenue à un niveau acceptable, relativement à la limite de dose, grâce à des conditions particulières d'humidité et de ventilation forcées qui y sont maintenues en permanence.

A côté du cas des mines d'uranium, l'exposition au radon et à ses produits de filiation radioactive existe dans toutes les galeries de mine, quel que soit le minerai extrait, car ce dernier présente toujours une radioactivité naturelle. Le charbon ne fait pas exception à cette règle au point que les effluents gazeux des centrales électriques au charbon présentent pour la population un risque radioactif (radionucléides surtout émetteurs $\alpha$ : uranium 234 et 238, thorium 230 et 232, radium 226,...) qui est du même ordre que celui présenté par les effluents gazeux radioactifs des centrales nucléaires (radionucléides émetteurs $\beta, \gamma:$ gaz rares, tritium, iode) $[6,8]$. Ces expositions sont de l'ordre du centième de l'exposition naturelle.

Pour ce qui concerne les efflents gazeux produits par les usines de retraitement des éléments combustibles irradiés dans les centrales nucléaires, les rejets conduisent à une exposition due essentiellement aux rayonnements $\beta, \gamma$ du krypton 85.

Pour une usine assurant le retraitement des éléments combustibles de 30 centrales nucléaires (environ 1000 t/an pour un combustible irradié à $30000 \mathrm{MWj} / \mathrm{t}$ ), l'exposition annuelle de l'organisme entier due au rayonnements $\gamma$ de $0,5 \mathrm{MeV}$ du krypton 85 serait, à proximité de l'usine, de quelques centièmes de l'exposition naturelle, soit quelques millièmes de la limite de dose ; l'irradiation de la peau par le rayonnement $\beta$ de 0,7 $\mathrm{MeV}$ du krypton 85 serait de quelques centièmes de la limite de dose pour la peau. Ces expositions sont encore relativement faibles et sont susceptibles d'être réduites, par piègeage du krypton sur charbon actif froid, d'un facteur voisin de 10 pour l'organisme entier et voisin de 100 pour la peau. 
Les effluents liquides de faible activité rejetés dans l'environnement par les usines de retraitement entraîneraient pour les personnes les plus exposées, celles qui vivent de leur pêche locale, une exposition qui serait au maximum de quelques centièmes de la limite de dose.

Les effluents liquides de très haute activité, résultant du retraitement des éléments combustibles irradiés et ayant subi une décroissance radioactive d'environ 10 années, sont transformés et stockés sous forme de blocs de verre qui assurent une bonne stabilité et dont le volume est d'environ $3 \mathrm{~m}^{3}$ par année de fonctionnement d'un réacteur fournissant une puissance électrique de $1 \mathrm{GW}$. Enfin, pour une même énergie électrique fournie, les déchets solides produits ont un volume d'environ $1200 \mathrm{~m}^{3}$; leur activité, faible, est due principalement à des radionucléides à courte période radioactive.

En conclusion, il est important de retenir que les effluents rejetés dans l'environnement sont toujours à un niveau d'activité faible en comparaison de la radioactivité naturelle et que les autres déchets sont stockés sous de très petits volumes et dans des conditions qui ne semblent pas susceptibles d'avoir des conséquences sanitaires, même dans un avenir éloigné.

\section{III.3. Pollution chimique}

Malgré les progrès technologiques réalisés pour l'extraction du charbon ou de tout minerai, le risque de pneumoconiose, résultant des poussières dans l'air de la mine, est annuellement de 0,7 \% de l'effectif ; il est, pour l'avenir, estimé à $4 \%$ pour une carrière de 30 ans [10]. Ce risque demeure donc important et il faut retenir que pour une même énergie électrique produite la masse de minerai à extraire de la mine, donc aussi le risque global qui en résulte, est environ 200 fois supérieure pour le charbon que pour un minerai d'uranium à $1 \%$.

Pour une puissance électrique de $1 \mathrm{GW}$, les polluants rejetés dans l'atmosphère par la cheminée d'une centrale utilisant du combustible fossile sont approximativement les suivants, sur la base d'une consommation annuelle de 2 Mt de fuel lourd (à $4 \%$ de soufre) ou $3 \mathrm{Mt}$ de charbon (à 2,3\% de soufre, et $13 \%$ de résidus solides imbrûlés) [12] :

\begin{tabular}{|c|c|c|c|c|}
\hline \multirow{2}{*}{$\begin{array}{l}\text { Polluant rejeté } \\
\text { en tonnes }\end{array}$} & \multicolumn{2}{|c|}{ en 1 jour } & \multicolumn{2}{|c|}{ en 1 an } \\
\hline & Fuel & Charbon & Fuel & Charbon \\
\hline $\begin{array}{l}\text { Oxydes de soufre } \\
\text { Oxydes d'azote } \\
\text { Hydrocarbure } \\
\text { Oxyde de carbone } \\
\text { Poussières } \\
\text { Gaz carbonique }\end{array}$ & $\begin{array}{c}440(1) \\
60 \\
2 \\
0,02 \\
1,2.10^{4}\end{array}$ & $\begin{array}{c}400(2) \\
60 \\
0,6 \\
1,4 \\
12(3) \\
3,1.10^{4}\end{array}$ & $\begin{array}{c}1,6.10^{5}(1) \\
2.10^{4} \\
700 \\
8 \\
4.10^{6}\end{array}$ & $\begin{array}{c}1,4.10^{5}(2) \\
2.10^{4} \\
200 \\
500 \\
4500 \\
1,1.10^{7}\end{array}$ \\
\hline \multicolumn{5}{|c|}{$\begin{array}{l}\text { (1) Essentiellement } \mathrm{SO}_{2} \\
\text { (2) Teneur très élevée en } \mathrm{SO}_{3} \\
\text { (3) Les cendres et les poussières retenues dans les filtres sont de } 1100 \text { t par jour, } \\
\text { soit } 4.10^{5} \text { t par an [1]. } \\
\text { Nota : Les charbons brûlés en France contiennent en moyenne } 0,7 \% \text { de soufre et } \\
\quad 15 \% \text { de résidus solides imbrûlés (ce dernier pourcentage s'élèverait à } 35 \% \\
\quad \text { si les charbons français étaient seuls utilisés [9]. }\end{array}$} \\
\hline
\end{tabular}


A priori, il est à craindre que tous ces effluents rejetés dans l'atmosphère par la combustion du fuel ou du charbon ne soient pas sans effet biologique sur les populations (bronchite chronique, cancers,...). L'augmentation de la hauteur des cheminées de rejet $(225 \mathrm{~m}$ par exemple pour la centrale au fuel de 2,8 GW implantée à Porcheville) n'apporte pas de diminution appréciable de la pollution de l'air au-delà de quelques kilomètres du point de rejet.

A côté de la pollution atmosphérique résultant de la consommation des combustibles fossiles, il est important d'attirer l'attention, et les écologistes le font rarement, sur le problème, peut-être important, de la teneur croissante de l'atmosphère en gaz carbonique. Malgré l'action stabilisatrice des océans et l'accroissement, fatalement lent, de la biomasse, la teneur en gaz carbonique de l'air est en augmentation constante [10] : $0,31 \%$ * en 1958, $0,33 \%$ en 1977 et environ $0,6 \%$ dans quelques décennies selon les estimations des spécialistes. Cet accroissement de gaz carbonique serait susceptible d'entraîner un accroissement de la température moyenne de la biosphère - en particulier d'environ $10^{\circ} \mathrm{C}$ dans les régions polaires. Dans ces conditions, ne peut-on pas craindre que le niveau des mers ne perde la stabilité relative observée depuis 5000 ans?

\section{FRÉQUENCES COMPARÉES DES DÉCÈS RÉSULTANT DES DIVERS MODES DE PRODUCTION D'ÉNERGIE}

Après le rappel de ces quelques éléments de comparaison, on comprendra plus facilement les résultats des estimations comparées des risques résultant des différents modes de production d'énergie [2, 3, 10].

Pour une même production d'énergie électrique, le nombre total des décès résultant à la fois de la pollution pour les travailleurs et la population et des accidents du travail est, suivant les évaluations faites, de 20 à 100 fois plus élevé du fait de l'utilisation du charbon, et à un moindre degré du pétrole, que du fait de l'utilisation de la réaction de fission (en retenant l'hypothèse pessimiste d'une relation dose-effet linéaire et sans seuil, voir 1.3.). Par contre, l'utilisation du gaz naturel conduit à un risque 3 fois moindre que la voie nucléaire (avec la même hypothèse). En fait, plus de $95 \%$ de ces décès résultent de la pollution atmosphérique dans le cas du charbon ou du pétrole et environ $50 \%$ dans le cas de l'uranium.

En définitive, pour une puissance électrique de $100 \mathrm{GW}$, le nombre annuel des décès par cancers, leucémies et autres maladies à évolution lente, suivant les diverses estimations faites [3], serait d'environ : 5 à 50 avec le combustible nucléaire utilisé seul (relation dose-effet linéaire), ou 100 à 10000 avec le combustible fossile utilisé seul (charbon ou pétrole).

\section{CONCLUSION}

Toute action ou inaction de l'homme comporte un risque. La notion de risque ne peut donc être que relative et son appréciation passe par une comparaison. L'usage du tabac qui constitue, aujourd'hui et pour longtemps encore, la cause principale de décès prématurés, peut servir de base

\footnotetext{
* en volume.
} 
de comparaison pour apprécier les dangers associés à la production d'énergie électrique : dans l'hypothèse d'une puissance électrique effective de 100 GW, la production d'électricité entraînerait, suivant les estimations précédentes et relativement aux 70000 décès annuels [11] résultant de l'usage du tabac en France (voir annexe) : 7 à 700 fois moins de décès que le tabagisme si le combustible fossile (gaz naturel excepté) était seul utilisé, 1400 à 14000 fois moins de décès que le tabagisme si le combustible nucléaire était seul utilisé (en retenant l'hypothèse pessimiste d'une relation dose-effet linéaire et sans seuil).

Pour la santé publique, l'objectif essentiel demeure la réduction du nombre important des décès précoces par tous les moyens de prévention possibles (par ordre d'importance décroissante) :

- limitation de l'usage du tabac (11\% des décès en 1976) : par la suppression de la distribution mensuelle de 320 cigarettes pour les jeunes appelés au service militaire, par l'exemple d'abstinence des éducateurs et des médecins, par l'extension des dispositions réglementaires de limitation déjà prises [5] mais encore insuffisantes, dans tous les lieux publics et dans tous les lieux de réunions;

- limitation de l'usage de l'alcool (6,5\% des décès) ;

- réduction du nombre des accidents de la route (2,5\% des décès) ;

- réduction du nombre des accidents domestiques et dans les loisirs (2\% des décès) ;

- réduction du nombre des maladies professionnelles et accidents du travail ( $1,4 \%$ des décès) ;

- amélioration de la filtration du rayonnement $X$ utilisé en radiodiagnostic et limitation de la section du faisceau utile à une surface contenue dans celle du film ;

- utilisation de la radiographie standard de préférence à la radiophotographie pour les contrôles radiologiques pulmonaires systématiques,

- utilisation de l'énergie nucléaire de préférence à l'énergie fossile (gaz naturel excepté) ; etc.

\section{ANNEXE}

\section{TABAGISME ET CANCER EN FRANCE}

Les 70000 décès annuels résultant de l'usage du tabac en France, dont environ 33000 par cancers, représentent $11,3 \%$ du total des décès ; ils correspondent donc à 6 millions de personnes concernées sur une population de 53 millions et ce nombre ne peut que croître dans les décennies à venir, surtout si le tabagisme féminin ne cesse lui aussi de croître.

Relativement à la fréquence des décès par cancers en 1976 (24,2\% pour les hommes et $18,4 \%$ pour les femmes), la fréquence des décès par cancers dus au tabagisme atteignait cette même année, $39 \%$ pour les hommes et 6,3\% pour les femmes.

La variation de la consommation du tabac entraîne une variation, dans le même sens, du taux de cancers mais avec un retard d'environ 20 années; c'est ainsi que pour les femmes, aux USA, le taux de cancers du poumon a été multiplié par 5 entre 1960 et 1980 par suite de l'accroissement de consommation entre 1940 et 1960. La même constatation est susceptible d'être observée en France, avec un décalage dans le temps par rapport aux USA. 


\section{BIBLIOGRAPHIE}

[1] BERTOZZI G., GOETZ L. Alpha-emitters from coal combustion, in comparison with a nuclear fuel cycle. ISPRA, Technical Note n 1.07.08.80.50, 1980.

[2] COHEN B.L., LEE I.S. A catalog of risks. Health Phys., 1979, 36, 707-722.

[3] COMAR C.L., SAGAN I.A. Health effects of energy production and conversion. Ann. Rev. Energy, 1976, 1, 581-589.

[4] Commission internationale de protection radiologique (CIPR). Recommandations (adoptées le 17 janv. 1977), publication CIPR 26, Oxford: Pergamon Press, 1978.

[5] Décret 77-1042 du 12 septembre 1977 relatif aux interdictions de fumer dans certains lieux affectés à un usage collectif. JORF, 17 septembre 1977, p. 4609.

[6] ILYIN L.A., KNIZHNIKOV V.A., BARKHUDAROV R.M. A relative risk estimation of excessive frequency of malignant tumors in population due to discharges into the atmosphere from fossil-fuel and nuclear power stations. In : IRPA 4 th international Congress, Paris, 24-30 April 1977, 1, 189-193.

[7] National academy of sciences, National research council, Comittee on the biological effects of ionizing radiation (BEIR). The effects on population of exposure to low levels of ionizing radiation (BEIR III). Washington: National academy press, 1980.

[8] Nations Unies. Comité scientifique des Nations Unies pour l'étude des effets des rayonnements ionisants (UNSCEAR). Sources et effets des rayonnements, rapport 1977. New-York: Nations Unies, 1978.

[9] ROLLIN Ph. Déchets et effluents des centrales thermiques classiques. In : Energie électrique et environnement, $5^{*}$ colloque, Villeneuve-lez-Avignon, 3-4 oct. 1980 (GINOCCHIO R., Ed.), 9-15.

[10] Société française d'énergie nucléaire (SFEN). Colloque sur les risques sanitaires des différentes énergies, Paris, 24-26 janvier 1980.

[11] TUBIANA M. Les bases biologiques de la radioprotection. Radioprotection, 1980 , $15,23-40$.

[12] WRIGHT J.H. Power and the environment. Westinghouse Electric International Company, Report 70-NE-18, 1970. 